\title{
STABLE PERIODIC ORBITS IN AN ELETROMECHANICAL SYSTEM
}

\author{
Márcio José Horta Dantas *, Rubens Sampaio ${ }^{\dagger}$, Roberta Lima $^{\dagger}$ \\ * Faculdade de Matemática \\ Universidade Federal de Uberlândia \\ 38400-902 Uberlândia M.G. , Brazil \\ $\dagger$ Mechanical Engineering Department, \\ PUC-Rio
}

Rua Marquês de São Vicente, 225; 38097, Rio de Janeiro, Brazil

Emails: marcio@ufu.br, rsampaio@puc-rio.br, roberta_ 


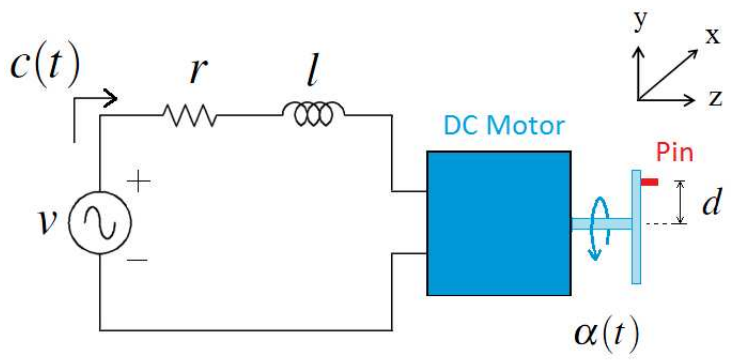

Figure 1: Electrical motor DC.

\subsection{Coupled cart-motor system: a master-slave relation}

As described in the introduction, the system analyzed in this paper is composed by a cart whose motion is driven by the DC motor sketched in Fig. 1. The motor is coupled to the cart through a pin that slides into a slot machined on an acrylic plate that is part to the cart, as shown in Fig. 2. The pin hole is drilled off-center on a disk fixed in the axis of the motor, so that the motor rotational motion is transformed into horizontal cart motion.

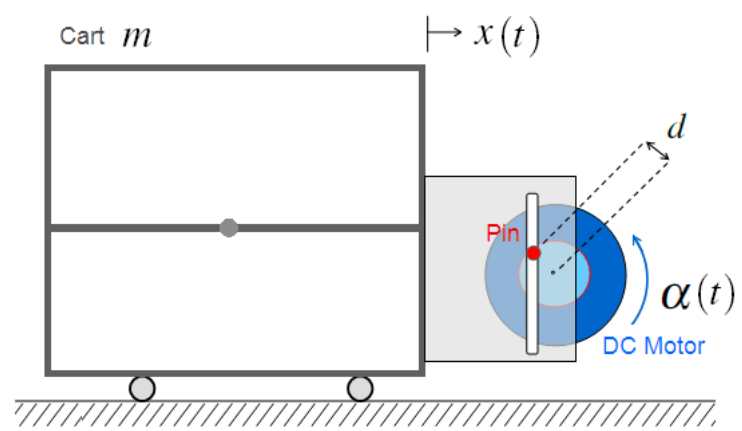

Figure 2: Coupled cart-motor system.

It is noticed that with this configuration, the center of mass of the mechanical system is always located in the center of mass of the cart, so its position does not change.

To model the coupling between the motor and the mechanical system, it is assumed that the motor shaft is rigid. Thus, the available torque to the coupled mechanical system, $\vec{\tau}$, can be written as

$$
\vec{\tau}(t)=\vec{d}(t) \times \vec{f}(t),
$$

where $\vec{d}$ is the eccentricity of the pin of the motor and $\vec{f}$ is the coupling force between the DC motor and the cart. By the problem geometry, the module of $\vec{d}$ is the nominal eccentricity of the pin, $d$. Besides this, the component of $\vec{d}$ that is perpendicular to the plane of the cart movement is always zero and, the others horizontal and verti- cal components can be calculated from the angular displacement $\alpha$ of the motor.

Assuming that there is no friction between the pin and the slot machined on an acrylic plate, the vector $\vec{f}$ only has a horizontal component, $f$ (the horizontal force that the DC motor exerts in the cart). Thus, $\vec{d}$ and $\vec{f}$ are written as

$$
\vec{d}(t)=\left[\begin{array}{c}
d \cos \alpha(t) \\
d \sin \alpha(t) \\
0
\end{array}\right] \quad \vec{f}(t)=\left[\begin{array}{c}
f(t) \\
0 \\
0
\end{array}\right]
$$

Substituting (3) (2), the available torque to the coupled mechanical system, $\vec{\tau}$, is

$$
\tau(t)=-f(t) d \sin \alpha(t)
$$

Due to constraints, the cart is not allowed to move in the vertical direction. Due to the problem geometry, the horizontal motion of the cart $x$ and the angular displacement $\alpha$ of the motor are related by the constraint

$$
x(t)=d \cos \alpha(t)
$$

Since the cart is modeled as a particle of mass $m$, it satisfies the equation

$$
m \ddot{x}(t)=f(t) .
$$

Substituting (4), (5) and (6) in the equations of the electric motor, we obtain a system of differential equations to the coupled system.

The initial value problem for the motor-cart system is: given the source voltage of the motor, $\nu$, find $(\alpha, c)$ satisfying

$$
\begin{aligned}
& l \dot{c}(t)+r c(t)+k_{e} \dot{\alpha}(t)=\nu, \\
& {\left[j_{m}+m d^{2}(\sin \alpha(t))^{2}\right] \ddot{\alpha}(t)} \\
& +\left[b_{m}+m d^{2} \dot{\alpha}(t) \cos \alpha(t) \sin \alpha(t)\right] \\
& \dot{\alpha}(t)-k_{t} c(t)=0,
\end{aligned}
$$

for given initial conditions.

Comparing (1) and (7) it is seen that the attached mass influences the motor in a parametric way, (Lacarbonara and Antman, 2008).

\section{The Problem}

Consider the system of (7). Taking $\dot{\alpha}(t)=u(t)$, the system can be written as a first order system, thus one gets that 


$$
\dot{c}(t)=-\frac{k_{e} u(t)+r c(t)-\nu}{}
$$


The other initial value problem is the following one 
the period of rotation of the disk, in the electromechanical system, is given by $\underline{2 \pi}$ 